\title{
Erratum to: In silico structural prediction of human steroid 5 $\alpha$-reductase type II
}

\author{
Wiranpat Karnsomwan ${ }^{1}$. Thanyada Rungrotmongkol ${ }^{2}$ - Wanchai De-Eknamkul ${ }^{1}$. \\ Supakarn Chamni ${ }^{1}$
}

Published online: 18 March 2017

(C) Springer Science+Business Media New York 2017

\section{Erratum to: Med Chem Res (2016) 25:1049-1056 DOI 10.1007/s00044-016-1541-y}

The original version of this article unfortunately contained a mistake. In Fig. 5, the structure of NADPH (3) was incorrect. The corrected Fig. 5 is given below.

Fig. 5 Structure of NADPH (3), finasteride (4), and dutasteride (5)

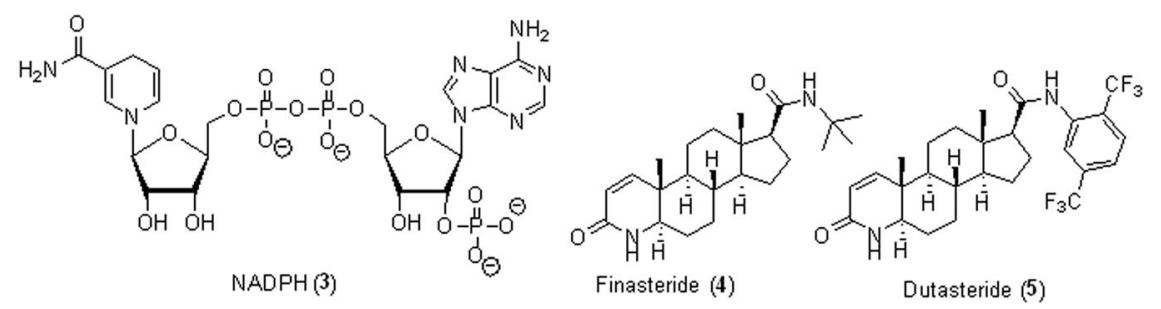

The online version of the original article can be found at doi:10.1007/ s00044-016-1541-y.

Supakarn Chamni

supakarn.c@pharm.chula.ac.th

Thanyada Rungrotmongkol

thanyada.r@chula.ac.th

Wanchai De-Eknamkul

dwanchai@chula.ac.th

1 Department of Pharmacognosy and Pharmaceutical Botany,

Faculty of Pharmaceutical Sciences, Chulalongkorn University,

Bangkok 10330, Thailand

2 Structural and Computational Biology Research Group,

Department of Biochemistry, Faculty of Science, Chulalongkorn

University, Bangkok 10330, Thailand 